\title{
PmLX66 and PmW14: New Alleles of Pm2 for Resistance to Powdery Mildew in the Chinese Winter Wheat Cultivars Liangxing 66 and Wennong 14
}

\author{
Yanling Sun, The National Key Facility for Crop Gene Resources and Genetic Improvement (NFCRI), Institute of Crop Sciences, Chinese \\ Academy of Agricultural Sciences, Beijing 100081; Jingwei Zou, Huigai Sun, and Wei Song, The National Key Facility for Crop Gene \\ Resources and Genetic Improvement (NFCRI), Institute of Crop Sciences, Chinese Academy of Agricultural Sciences, Beijing 100081; and \\ College of Life Science and Technology, Hebei Normal University of Science and Technology, Qinhuangdao 066004, China; and Xiaoming \\ Wang and Hongjie Li, The National Key Facility for Crop Gene Resources and Genetic Improvement (NFCRI), Institute of Crop Sciences, \\ Chinese Academy of Agricultural Sciences, Beijing 100081
}

\begin{abstract}
Sun, Y., Zou, J., Sun, H., Song, W., Wang, X., and Li, H. 2015. PmLX66 and PmW14: New alleles of Pm2 for resistance to powdery mildew in the Chinese winter wheat cultivars Liangxing 66 and Wennong 14. Plant Dis. 99:1118-1124.

Wheat powdery mildew (caused by Blumeria graminis f. sp. tritici) can be effectively managed by growing resistant cultivars. 'Liangxing 66 ' and 'Wennong 14' are the current winter wheat cultivars grown in northern China where powdery mildew is epidemic. Both cultivars have been demonstrated to carry single dominant genes for resistance to powdery mildew, tentatively designated PmLX66 and PmW14, on chromosome 5DS and share common linked markers with $P m 2$. Allelism tests were performed using a total of 15,657 plants of $\mathrm{F}_{2}$ segregating populations to determine the relationship between PmLX66, PmW14, and Pm2. All progeny from the crosses Liangxing $66 \times$ 'Ulka/ $8 *$ Chancellor' $(\mathrm{Ulka} / 8 * \mathrm{Cc})$,

Wennong $14 \times \mathrm{Ulka} / 8 * \mathrm{Cc}$, and Liangxing $66 \times$ Wennong 14 were resistant when tested with $B$. graminis $\mathrm{f}$. $\mathrm{sp}$. tritici isolate E20, indicating that $P m L X 66$ and PmW14 are allelic to Pm2 and to each other. Liangxing 66 was resistant to $76.7 \%$ of the $60 \mathrm{~B}$. graminis $\mathrm{f}$. sp. tritici isolates from northern China, a slightly smaller proportion than Ulka/8*Cc $(78.3 \%)$. However, Wennong $14(85.0 \%)$ was more resistant against this set of B. graminis f. sp. tritici isolates than $\mathrm{Ulka} / 8 * \mathrm{Cc}$ and Liangxing 66 . Liangxing 66 and Wennong 14 differed from Ulka/ $8 * \mathrm{Cc}$ in respect to a number of $B$. graminis f. sp. tritici isolates. Based on these findings, $P m L X 66$ and PmW14 are new alleles at the Pm2 locus.
\end{abstract}

Powdery mildew, caused by the biotrophic fungus Blumeria graminis f. sp. tritici, is a major constraint to common wheat (Triticum aestivum L.) production throughout the world, especially in the wheat-producing regions with maritime or semicontinental climates (Cowger et al. 2012). Wheat production in China has been suffering from this destructive disease since the 1970s. Currently, the area affected by powdery mildew in China ranges from 6 to 8 million ha, which encompasses nearly all the major wheat-producing regions of the country, with more severe damage in the winter- than springwheat regions (http://cb.natesc.gov.cn).

Losses in yield and end-use quality caused by powdery mildew can be limited by fungicides but the use of resistant cultivars is the preferred way to reduce the effects of epidemics and minimize yield losses (Hulbert et al. 2001). Reduced input cost for farmers is another benefit of growing powdery mildew-resistant cultivars.

Availability of powdery mildew resistance genes $(\mathrm{Pm})$ is a prerequisite for developing resistant cultivars. Over 60 genes conferring qualitative resistance to wheat powdery mildew have been mapped (http://www.shigen.nig.ac.jp/wheat/komugi/genes/symbolListPage Action.do?page =-1). Most loci contain a single resistance allele but some (e.g., Pm1, Pm3, Pm4, Pm5, Pm8, and Pm24) have multiple alleles (Hsam and Zeller 1997; Huang et al. 2003; Schmolke et al. 2012; Singrün et al. 2003; Xue et al. 2012; Yahiaoui et al. 2009). These genes are race specific, short lived, and frequently overcome by virulent pathotypes. Genes $P m 6$ and $P m 8$, for example, were widely deployed in commercial wheat cultivars or in combinations with other powdery mildew resistance genes in China (Li et al. 2011;

Present address of W. Song: Hunan University, Changsha 410082, China.

Corresponding author: H. Li; E-mail: lihongjie@caas.cn

Accepted for publication 19 December 2014.

http://dx.doi.org/10.1094/PDIS-10-14-1079-RE

(C) 2015 The American Phytopathological Society
Sang et al. 2006; Zhan et al. 2010) and other countries (Purnhauser et al. 2011; Rabinovich 1998). The results of a recent study using 1,082 B. graminis f. sp. tritici isolates collected from different wheatproduction regions of China demonstrated that the majority of isolates (>70\%) were virulent on Pm6 and Pm8 (Zeng et al. 2014). In contrast, the number of $B$. graminis $\mathrm{f}$. sp. tritici isolates that are virulent on Pm2 remained low in some regions of China and other parts of the world (Cao et al. 2010; Lutz et al. 1992, 1995; Zhou et al. 2002), although Pm2 was identified several decades ago (Pugsley and Carter 1953).

The use of a specific powdery mildew resistance gene depends on its effectiveness and the agronomic performance of wheat cultivars containing it. Therefore, not all identified genes are easily used in developing cultivars due to linkage drag (Summers and Brown 2013). 'Liangxing 66' and 'Wennong 14' are commercial winter wheat cultivars that have broad-spectrum resistance to powdery mildew in northern China. Resistance in these cultivars was reportedly controlled by single dominant genes tentatively designated PmLX66 and PmW14 on chromosome 5DS, on which Pm2 is located (Huang et al. 2012; Song et al. 2014).

As a follow-up study, the present allelism test was conducted using large segregating populations derived from the crosses between Liangxing 66, Wennong 14 , and 'Ulka/8*Chancellor' (Ulka/8* Cc), a near-isogenic line with known $P m 2$ produced by repeatedly backcrossing 'Ulka' with the recurrent parent 'Chancellor' (Briggle 1969). The reactions of Liangxing 66 and Wennong 14 to additional B. graminis f. sp. tritici isolates collected from northern China were examined. The objectives of this study were to elucidate the relationship between PmLX66, PmW14, and Pm2 and determine the effectiveness of these newly identified genes.

\section{Materials and Methods}

Plant materials. Winter wheat Liangxing 66, developed from the cross 'Ji 91102' × 'Ji 935031' by the Shandong Liangxing Seed Co. Ltd., Ningjin, Shandong, has been commercialized in a wide region of Shandong, Hebei, Shanxi, Henan, Anhui, Jiangsu, and Shaanxi provinces and Tianjin across the Yellow and Huai River Valleys winter wheat zone and the northern winter wheat zone (http://www. cnpvp.com/). Wennong 14, a winter wheat cultivar produced by 
Table 1. Infection types produced on seedlings of Liangxing 66 (PmLX66), Wennong 14, Ulka/8*Cc (Pm2), and Tabasco (Pm48) and virulence frequencies on different known powdery mildew resistance genes of a set of Blumeria graminis f. sp. tritici isolates originating from different wheat-producing regions of China

\begin{tabular}{|c|c|c|c|c|c|c|c|c|c|}
\hline Isolates & Origin & $\begin{array}{l}\text { Avirulent/virulent } \\
\text { on } P m \text { genes }^{\mathbf{z}}\end{array}$ & $\begin{array}{c}\text { Liangxing } 66 \\
(\text { PmLX66) }\end{array}$ & $\begin{array}{l}\text { Wennong } 14 \\
(\text { PmW14 })\end{array}$ & $\begin{array}{l}\text { Ulka/8*Cc } \\
(\text { Pm2 })\end{array}$ & $\begin{array}{c}\text { Tabasco } \\
(P m 48)\end{array}$ & Chancellor & $\begin{array}{c}\text { Zhongzuo } \\
9504\end{array}$ & $\begin{array}{c}\text { Virulence } \\
\text { frequency }(\%)\end{array}$ \\
\hline $\mathrm{Bg} 44-4$ & $\begin{array}{l}\text { Zhanhua, } \\
\text { Shandong }\end{array}$ & $\begin{array}{l}1 \mathrm{c}, 2,3,3 \mathrm{~b}, 3 \mathrm{~g}, 4 \mathrm{a}, 4 \mathrm{~b}, 5 \mathrm{e}, 6,13,21,24, \\
33 / 1 \mathrm{a}, 3 \mathrm{c}, 5 \mathrm{a}, 8,19,46\end{array}$ & 3 & 3 & 0 & 3 & 3 & 4 & 31.6 \\
\hline $\mathrm{Bg} 44-5$ & $\begin{array}{l}\text { Zhanhua, } \\
\text { Shandong }\end{array}$ & $\begin{array}{r}\text { 1a,1c,4b,5e, } 13,17,21,24,33 / 2 \\
3,3 \mathrm{~b}, 3 \mathrm{c}, 3 \mathrm{~g}, 4 \mathrm{a}, 5 \mathrm{a}, 6,7,8,19,46\end{array}$ & 3 & 3 & 3 & 3 & 3 & 3 & 57.1 \\
\hline $\mathrm{Bg} 44-6$ & $\begin{array}{l}\text { Zhanhua, } \\
\text { Shandong }\end{array}$ & $\begin{array}{c}1 \mathrm{a}, 1 \mathrm{c}, 4 \mathrm{a}, 4 \mathrm{~b}, 5 \mathrm{e}, 13,19,21,24,33 \\
46 / 2,3,3 \mathrm{~b}, 3 \mathrm{c}, 3 \mathrm{~g}, 5 \mathrm{a}, 6,8\end{array}$ & 3 & 3 & 3 & 1 & 3 & 3 & 42.1 \\
\hline Bg79-1 & $\begin{array}{l}\text { Jining, } \\
\text { Shandong }\end{array}$ & $\begin{array}{c}\text { 1c,5e,13,17,21,24,33/1a, } 2,3,3 \mathrm{~b} \\
3 \mathrm{c}, 3 \mathrm{~g}, 4 \mathrm{a}, 4 \mathrm{~b}, 5 \mathrm{a}, 7,8,19,46\end{array}$ & 3 & 3 & 3 & 3 & 3 & 4 & 65.0 \\
\hline $\mathrm{Bg} 79-2$ & $\begin{array}{l}\text { Jining, } \\
\text { Shandong }\end{array}$ & $\begin{array}{c}1 \mathrm{c}, 2,5 \mathrm{e}, 8,13,21,24,33,46 / 1 \mathrm{a}, 3,3 \mathrm{~b}, \\
3 \mathrm{c}, 3 \mathrm{~g}, 4 \mathrm{a}, 4 \mathrm{~b}, 5 \mathrm{a}, 6,19\end{array}$ & 3 & 3 & 0 & 0 & 3 & 4 & 52.6 \\
\hline $\mathrm{Bg} 79-3$ & $\begin{array}{l}\text { Jining, } \\
\text { Shandong }\end{array}$ & $\begin{array}{l}1 \mathrm{a}, 1 \mathrm{c}, 2,3 \mathrm{~b}, 4 \mathrm{a}, 4 \mathrm{~b}, 5 \mathrm{e} \\
6,13,19,21,24,46 / 3,3 \mathrm{c} \\
3 \mathrm{~g}, 5 \mathrm{a}, 7,8,17,33\end{array}$ & 3 & 3 & 0 & 0 & 3 & 3 & 38.1 \\
\hline $\mathrm{Bg} 80-3$ & $\begin{array}{l}\text { Juxian, } \\
\text { Shandong }\end{array}$ & $\begin{array}{l}\text { 1a, } 1 \mathrm{c}, 2,3 \mathrm{~b}, 5 \mathrm{a}, 5 \mathrm{e} \\
13,17,21,24,46 / 3,3 \mathrm{c}, 3 \mathrm{~g} \\
4 \mathrm{a}, 4 \mathrm{~b}, 7,8,19,33\end{array}$ & 0 & 0 & 0 & 1 & 3 & 3 & 45.0 \\
\hline Bg81-2 & $\begin{array}{l}\text { Pingyi, } \\
\text { Shandong }\end{array}$ & $\begin{array}{l}\text { 1a, } 1 \mathrm{c}, 2,5 \mathrm{e}, 13,21,24 \\
33,46 / 3,3 \mathrm{~b}, 3 \mathrm{c}, 3 \mathrm{~g}, 4 \mathrm{a} \\
\text { 4b,5a,6,7,8,17,19 }\end{array}$ & 0 & 0 & 0 & 0 & 3 & 3 & 57.1 \\
\hline $\mathrm{Bg} 81-3$ & $\begin{array}{l}\text { Pingyi, } \\
\text { Shandong }\end{array}$ & $\begin{array}{l}1 \mathrm{c}, 2,5 \mathrm{e}, 13,17,21,24,46 / 1 \mathrm{a}, \\
3,3 \mathrm{~b}, 3 \mathrm{c}, 3 \mathrm{~g}, 4 \mathrm{a}, 4 \mathrm{~b} \\
5 \mathrm{a}, 7,8,19,33\end{array}$ & 0 & 0 & 0 & 0 & 3 & 4 & 60.0 \\
\hline Bg82-1 & $\begin{array}{l}\text { Shoushan, } \\
\text { Shandong }\end{array}$ & $\begin{array}{l}\text { 1c,5e, }, 8,13,21,24,46 / \\
1 \mathrm{a}, 2,3,3 \mathrm{~b}, 3 \mathrm{c}, 3 \mathrm{~g} \\
\text { 4a,4b,5a,6,7,17,19,33 }\end{array}$ & 3 & 0 & 4 & 0 & 3 & 4 & 66.7 \\
\hline $\mathrm{Bg} 82-2$ & $\begin{array}{l}\text { Shoushan, } \\
\text { Shandong }\end{array}$ & $\begin{array}{l}\text { 1c, } 2,5 \mathrm{e}, 6,8,13,17,21, \\
24,33,46 / 1 \mathrm{a}, 3,3 \mathrm{~b}, 3 \mathrm{c}, 3 \mathrm{~g}, 4 \mathrm{a}, \\
4 \mathrm{~b}, 5 \mathrm{a}, 7,19\end{array}$ & 0 & 0 & 0 & 0 & 3 & 3 & 47.6 \\
\hline $\mathrm{Bg} 82-3$ & $\begin{array}{l}\text { Shoushan, } \\
\text { Shandong }\end{array}$ & $\begin{array}{l}1 \mathrm{c}, 2,3,3 \mathrm{~b}, 3 \mathrm{c}, 3 \mathrm{~g}, 4 \mathrm{a}, 4 \mathrm{~b}, 5 \mathrm{a} \\
5 \mathrm{e}, 6,13,17,21,24 \\
33,46 / 1 \mathrm{a}, 8,19\end{array}$ & 0 & 0 & 0 & 0 & 3 & 3 & 14.3 \\
\hline Bg83-1 & $\begin{array}{l}\text { Wendeng, } \\
\text { Shandong }\end{array}$ & $\begin{array}{r}1 \mathrm{c}, 3 \mathrm{~b}, 3 \mathrm{~g}, 4 \mathrm{a}, 4 \mathrm{~b}, 5 \mathrm{e}, 6 \\
13,17,21,24,33,46 / \\
1 \mathrm{a}, 2,3,3 \mathrm{c}, 5 \mathrm{a}, 7,8,19\end{array}$ & 3 & 0 & 3 & 0 & 3 & 3 & 38.1 \\
\hline $\mathrm{Bg} 83-2$ & $\begin{array}{l}\text { Wendeng, } \\
\text { Shandong }\end{array}$ & $\begin{array}{c}1 \mathrm{c}, 3 \mathrm{~b}, 3 \mathrm{c}, 4 \mathrm{a}, 4 \mathrm{~b}, 5 \mathrm{a}, 5 \mathrm{e} \\
8,13,17,21,24,33 / \\
1 \mathrm{a}, 2,3,3 \mathrm{~g}, 7,19,46\end{array}$ & 3 & 0 & 3 & 3 & 3 & 3 & 35.0 \\
\hline Bg84-1 & $\begin{array}{l}\text { Yuncheng, } \\
\text { Shandong }\end{array}$ & $\begin{array}{l}1 \mathrm{c}, 3,3 \mathrm{~b}, 4 \mathrm{a}, 4 \mathrm{~b}, 8,13 \\
17,21,24,33,46 / \\
1 \mathrm{a}, 2,3 \mathrm{c}, 3 \mathrm{~g}, 5 \mathrm{a}, 5 \mathrm{e}, 6,7,19\end{array}$ & 1 & 3 & 3 & 0 & 3 & 4 & 42.9 \\
\hline $\mathrm{Bg} 84-2$ & $\begin{array}{l}\text { Yuncheng, } \\
\text { Shandong }\end{array}$ & $\begin{array}{l}1 \mathrm{a}, 1 \mathrm{c}, 2,3 \mathrm{~b}, 5 \mathrm{e}, 13 \\
21,24,46 / 3,3 \mathrm{c}, 3 \mathrm{~g}, 4 \mathrm{a} \\
4 \mathrm{~b}, 5 \mathrm{a}, 6,7,8,17,19,33\end{array}$ & 0 & 0 & 0 & 0 & 4 & 4 & 57.1 \\
\hline Bg84-3 & $\begin{array}{l}\text { Yuncheng, } \\
\text { Shandong }\end{array}$ & $\begin{array}{l}\text { 1a, } 1 \mathrm{c}, 3 \mathrm{~b}, 5 \mathrm{e}, 13,17,21 \\
24,33,46 / 2,3,3 \mathrm{c}, 3 \mathrm{~g}, 4 \mathrm{a} \\
\text { 4b,5a,7,8,19 }\end{array}$ & 3 & 0 & 3 & 0 & 3 & 3 & 50.0 \\
\hline Bg85-1 & $\begin{array}{l}\text { Zhaoyuan, } \\
\text { Shandong }\end{array}$ & $\begin{array}{l}\text { 1a, } 1 \mathrm{c}, 2,3,4 \mathrm{a}, 4 \mathrm{~b}, 21,24, \\
33,46 / 3 \mathrm{~b}, 3 \mathrm{c}, 3 \mathrm{~g}, 5 \mathrm{a}, 5 \mathrm{e}, 6 \\
7,8,13,17,19\end{array}$ & 1 & 0 & 2 & 0 & 4 & 3 & 52.4 \\
\hline Bg85-2 & $\begin{array}{l}\text { Zhaoyuan, } \\
\text { Shandong }\end{array}$ & $\begin{array}{l}\text { 1a, } 1 \mathrm{c}, 2,4 \mathrm{~b}, 13,17,21 \\
24,33,46 / 3,3 \mathrm{~b}, 3 \mathrm{c}, 3 \mathrm{~g} \\
\text { 4a,5a,5e,7,8,19 }\end{array}$ & 0 & 0 & 0 & 0 & 3 & 4 & 50.0 \\
\hline $\mathrm{Bg} 85-3$ & $\begin{array}{l}\text { Zhaoyuan, } \\
\text { Shandong }\end{array}$ & $\begin{array}{l}\text { 1c, } 2,3,4 \mathrm{a}, 4 \mathrm{~b}, 13,21,24 \\
33,46 / 1 \mathrm{a}, 3 \mathrm{~b}, 3 \mathrm{c}, 3 \mathrm{~g}, 5 \mathrm{a}, 5 \mathrm{e} \\
6,7,8,17,19\end{array}$ & 0 & 0 & 0 & 0 & 4 & 4 & 52.4 \\
\hline $\mathrm{Bg} 88-2$ & $\begin{array}{l}\text { Haiyang, } \\
\text { Shandong }\end{array}$ & $\begin{array}{l}\text { 1c,3b,5e,21,24,46/1a,2,3,3c, } \\
\text { 3g,4a,4b,5a, } \\
6,7,8,13,17,19,33\end{array}$ & 0 & 0 & 3 & 0 & 3 & 4 & 71.4 \\
\hline Bg88-3 & $\begin{array}{l}\text { Haiyang, } \\
\text { Shandong }\end{array}$ & $\begin{array}{l}\text { 1a, } 1 \mathrm{c}, 2,3 \mathrm{~b}, 5 \mathrm{e}, 13,21 \\
24,46 / 3,3 \mathrm{c}, 3 \mathrm{~g}, 4 \mathrm{a}, 4 \mathrm{~b} \\
5 \mathrm{a}, 6,7,8,17,19,33\end{array}$ & 1 & 0 & 0 & 0 & 3 & 4 & 57.1 \\
\hline Bg57-3 & $\begin{array}{l}\text { Xingtai, } \\
\text { Hebei }\end{array}$ & $\begin{array}{l}\text { 1a,1c, } 2,3,5 \mathrm{e}, 21,24, \\
\text { 46/3b,3c,3g,4a,4b,5a,6,7, } \\
8,13,17,19,33\end{array}$ & 0 & 0 & 0 & 0 & 4 & 4 & 61.9 \\
\hline Bg57-4 & $\begin{array}{c}\text { Xingtai, } \\
\text { Hebei }\end{array}$ & $\begin{array}{l}1 \mathrm{c}, 2,3,5 \mathrm{e}, 6,13,17,21 \\
24,46 / 1 \mathrm{a}, 3 \mathrm{~b}, 3 \mathrm{c}, 3 \mathrm{~g}, 4 \mathrm{a}, 4 \mathrm{~b} \\
5 \mathrm{a}, 7,8,19,33\end{array}$ & 0 & 0 & 0 & 0 & 4 & 4 & 52.4 \\
\hline
\end{tabular}

z 1 = Festival, Pmla; $2=P m 1 c ; 3=\mathrm{Ulka} / 8 * \mathrm{Cc}, P m 2 ; 4=+P m 3 a ; 5=\mathrm{CI} 14121, P m 3 b ; 6=$ Sonora $/ 8 * \mathrm{Cc}, P m 3 c ; 7=\mathrm{Courtot}, P m 3 g ; 8=\mathrm{Khaphi} / 8 * \mathrm{Cc}, P m 4 a ; 9=$ VPM1, Pm4b; $10=$ CI 14125, Pm5a; $11=$ Fuzhuang 30, Pm5e; $12=$ Coker 747, Pm6; $13=$ CI 141879, Pm7; $14=$ PI 361879, Pm8; $15=96-282$, Pm13; $16=$ Amigo, $P m 17 ; 17=$ 96-286, Pm19; 18 = R77/6*Bainong 3217, Pm21; 19 = Chiyacao, $P m 24 ; 20=2636-32 \mathrm{R}, P m 33 ; 21=$ Tabasco, $P m 48$. 
Table 1. (continued from preceding page)

\begin{tabular}{|c|c|c|c|c|c|c|c|c|c|}
\hline Isolates & Origin & $\begin{array}{l}\text { Avirulent/virulent } \\
\text { on } P m \text { genes }^{z}\end{array}$ & $\begin{array}{l}\text { Liangxing } 66 \\
\text { (PmLX66) }\end{array}$ & $\begin{array}{l}\text { Wennong } 14 \\
\quad(P m W 14)\end{array}$ & $\begin{array}{l}\text { Ulka/8*Cc } \\
\quad(P m 2)\end{array}$ & $\begin{array}{l}\text { Tabasco } \\
(P m 48)\end{array}$ & Chancellor & $\begin{array}{c}\text { Zhongzuo } \\
9504\end{array}$ & $\begin{array}{c}\text { Virulence } \\
\text { frequency }(\%)\end{array}$ \\
\hline Bg57-5 & $\begin{array}{c}\text { Xingtai, } \\
\text { Hebei }\end{array}$ & $\begin{array}{l}1 \mathrm{a}, 1 \mathrm{c}, 2,4 \mathrm{a}, 4 \mathrm{~b}, 5 \mathrm{e}, 8,13 \\
17,21,24,33 / \\
3,3 \mathrm{~b}, 3 \mathrm{c}, 3 \mathrm{~g}, 5 \mathrm{a}, 7,19,46\end{array}$ & 0 & 0 & 0 & 3 & 3 & 4 & 40.0 \\
\hline Bg69-1 & $\begin{array}{l}\text { Cixian, } \\
\text { Hebei }\end{array}$ & $\begin{array}{l}\text { 1a,1c, } 2,3 \mathrm{~b}, 3 \mathrm{c}, 4 \mathrm{~b}, 5 \mathrm{e}, 13,21,24,33 \\
\quad 46 / 3,3 \mathrm{~g}, 4 \mathrm{a}, 5 \mathrm{a}, 6,7,8,19\end{array}$ & 0 & 0 & 0 & 0 & 3 & 4 & 40.0 \\
\hline Bg69-2 & $\begin{array}{l}\text { Cixian, } \\
\text { Hebei }\end{array}$ & $\begin{array}{l}1 \mathrm{a}, 1 \mathrm{c}, 2,3 \mathrm{~b}, 3 \mathrm{c}, 5 \mathrm{e}, 13 \\
17,21,24,46 / 3,3 \mathrm{~g}, 4 \mathrm{a} \\
\text { 4b,5a,7,8,19,33 }\end{array}$ & 0 & 0 & 0 & 0 & 3 & 4 & 50.0 \\
\hline Bg69-3 & $\begin{array}{l}\text { Cixian, } \\
\text { Hebei }\end{array}$ & $\begin{array}{l}1 \mathrm{a}, 1 \mathrm{c}, 2,3 \mathrm{~b}, 3 \mathrm{~g}, 5 \mathrm{a} \\
5 \mathrm{e}, 8,13,17,21,24 \\
33,46 / 3,3 \mathrm{c}, 4 \mathrm{a}, 7,19\end{array}$ & 0 & 0 & 0 & 0 & 3 & 4 & 31.6 \\
\hline $\mathrm{Bg} 70-1$ & $\begin{array}{l}\text { Gucheng, } \\
\text { Hebei }\end{array}$ & $\begin{array}{l}\text { 1a, } 1 \mathrm{c} 13,21,24 \\
\text { 46/2,3,3b,3c,3g } \\
\text { 4a,4b,5a,5e,6} \\
7,8,17,19,33\end{array}$ & 0 & 0 & 4 & 0 & 4 & 4 & 71.4 \\
\hline Bg70-2 & $\begin{array}{l}\text { Gucheng, } \\
\text { Hebei }\end{array}$ & $\begin{array}{l}\text { 1a,1c, } 2,13,21,24,33 \\
\text { 46/3,3b,3c,3g,4a,4b,5a, } \\
\text { 5e,6,7,8,17,19 }\end{array}$ & 0 & 0 & 0 & 0 & 4 & 4 & 66.7 \\
\hline $\mathrm{Bg} 70-3$ & $\begin{array}{l}\text { Gucheng, } \\
\text { Hebei }\end{array}$ & $\begin{array}{l}1 \mathrm{a}, 1 \mathrm{c}, 2,5 \mathrm{e}, 21,24 \\
46 / 3,3 \mathrm{~b}, 3 \mathrm{c}, 3 \mathrm{~g}, 4 \mathrm{a}, 4 \mathrm{~b}, 5 \mathrm{a} \\
6,7,8,13,17,19,33\end{array}$ & 0 & 0 & 0 & 0 & 4 & 4 & 71.4 \\
\hline Bg70-? & $\begin{array}{l}\text { Gucheng, } \\
\text { Hebei }\end{array}$ & $\begin{array}{l}1 \mathrm{a}, 1 \mathrm{c}, 2,5 \mathrm{e}, 13,21 \\
24 / 3,3 \mathrm{~b}, 3 \mathrm{c}, 3 \mathrm{~g}, 4 \mathrm{a}, 4 \mathrm{~b} \\
5 \mathrm{a}, 7,8,17,19,33,46\end{array}$ & 0 & 0 & 0 & 3 & 3 & 4 & 70.0 \\
\hline $\mathrm{Bg} 71-2$ & $\begin{array}{l}\text { Shahe, } \\
\text { Hebei }\end{array}$ & $\begin{array}{l}1 \mathrm{a}, 1 \mathrm{c}, 2,5 \mathrm{e}, 6,13 \\
21,24,46 / 3,3 \mathrm{~b}, 3 \mathrm{c}, 3 \mathrm{~g} \\
4 \mathrm{a}, 4 \mathrm{~b}, 5 \mathrm{a}, 8,19,33\end{array}$ & 0 & 0 & 0 & 0 & 3 & 3 & 52.6 \\
\hline Bg71-3 & $\begin{array}{l}\text { Shahe, } \\
\text { Hebei }\end{array}$ & $\begin{array}{l}\text { 1a,1c, } 2,6,13,21,24 \\
46 / 3,3 \mathrm{~b}, 3 \mathrm{c}, 3 \mathrm{~g}, 4 \mathrm{a}, 4 \mathrm{~b}, 5 \mathrm{a} \\
5 \mathrm{e}, 7,8,17,19,33\end{array}$ & 0 & 0 & 0 & 0 & 4 & 4 & 61.9 \\
\hline Bg72 & $\begin{array}{l}\text { Shijiazhuang, } \\
\text { Hebei }\end{array}$ & $\begin{array}{l}1 \mathrm{a}, 1 \mathrm{c}, 2,3 \mathrm{~b}, 4 \mathrm{a}, 4 \mathrm{~b}, 5 \mathrm{e} \\
6,21,24,33,46 / 3,3 \mathrm{c} \\
3 \mathrm{~g}, 5 \mathrm{a}, 8,19\end{array}$ & 0 & 0 & 0 & 0 & 3 & 3 & 33.3 \\
\hline Bg73-2 & $\begin{array}{l}\text { Yuanshi, } \\
\text { Hebei }\end{array}$ & $\begin{array}{l}\text { 1a,1c,2,3,5e,21,24,46/ } \\
\text { 3b,3c,3g,4a,4b,5a,6,7,8, } \\
13,17,19,33\end{array}$ & 0 & 0 & 0 & 0 & 4 & 4 & 61.9 \\
\hline $\mathrm{Bg} 73-3$ & $\begin{array}{l}\text { Yuanshi, } \\
\text { Hebei }\end{array}$ & $\begin{array}{l}1 \mathrm{a}, 1 \mathrm{c}, 2,4 \mathrm{~b}, 5 \mathrm{e}, 6,13 \\
19,21,24,46 / 3,3 \mathrm{~b}, 3 \mathrm{c}, 3 \mathrm{~g} \\
4 \mathrm{a}, 5 \mathrm{a}, 8,33\end{array}$ & 0 & 0 & 0 & 0 & 3 & 3 & 47.4 \\
\hline Bg74-1 & $\begin{array}{l}\text { Zhuozhou, } \\
\text { Hebei }\end{array}$ & $\begin{array}{l}1 \mathrm{a}, 1 \mathrm{c}, 2,3 \mathrm{~g}, 4 \mathrm{a}, 4 \mathrm{~b}, 5 \mathrm{e}, 13 \\
21,24,33,46 / 3,3 \mathrm{~b}, 3 \mathrm{c}, 5 \mathrm{a} \\
6,7,8,19\end{array}$ & 0 & 0 & 0 & 0 & 3 & 3 & 40.0 \\
\hline Bg74-2 & $\begin{array}{l}\text { Zhuozhou, } \\
\text { Hebei }\end{array}$ & $\begin{array}{l}\text { 1a,1c, } 2,4 \mathrm{~b}, 5 \mathrm{e}, 13,17,21 \\
24,33,46 / 3,3 \mathrm{~b}, 3 \mathrm{c}, 3 \mathrm{~g}, 4 \mathrm{a} \\
\text { 5a,7,8,19 }\end{array}$ & 0 & 0 & 0 & 0 & 3 & 4 & 45.0 \\
\hline $\mathrm{Bg} 74-3$ & $\begin{array}{l}\text { Zhuozhou, } \\
\text { Hebei }\end{array}$ & $\begin{array}{l}1 \mathrm{a}, 1 \mathrm{c}, 2,3,4 \mathrm{a}, 4 \mathrm{~b}, 5 \mathrm{e} \\
13,21,24,33,46 / 3 \mathrm{~b}, 3 \mathrm{c}, 3 \mathrm{~g} \\
\text { 5a,6,7,8,19 }\end{array}$ & 1 & 0 & 0 & 0 & 3 & 4 & 42.9 \\
\hline Bg75-1 & $\begin{array}{l}\text { Xunxian, } \\
\text { Henan }\end{array}$ & $\begin{array}{l}1 \mathrm{a}, 1 \mathrm{c}, 2,3 \mathrm{~b}, 5 \mathrm{e}, 13,17,21 \\
24,46 / 3,3 \mathrm{c}, 3 \mathrm{~g}, 4 \mathrm{a}, 4 \mathrm{~b}, 5 \mathrm{a} \\
6,8,19,33\end{array}$ & 0 & 0 & 0 & 0 & 3 & 4 & 47.6 \\
\hline Bg75-2 & $\begin{array}{l}\text { Xunxian, } \\
\text { Henan }\end{array}$ & $\begin{array}{l}1 \mathrm{a}, 1 \mathrm{c}, 2,5 \mathrm{e}, 8,13,21,24 \\
46 / 3,3 \mathrm{~b}, 3 \mathrm{c}, 3 \mathrm{~g}, 4 \mathrm{a}, 4 \mathrm{~b}, 5 \mathrm{a} \\
6,7,19,33\end{array}$ & 0 & 0 & 0 & 0 & 3 & 3 & 60.0 \\
\hline $\mathrm{Bg} 75-3$ & $\begin{array}{l}\text { Xunxian, } \\
\text { Henan }\end{array}$ & $\begin{array}{l}2,3 \mathrm{~b}, 5 \mathrm{e}, 13,21,24 \\
\text { 46/1a, } 1 \mathrm{c}, 3,3 \mathrm{c}, 3 \mathrm{~g}, 4 \mathrm{a}, 4 \mathrm{~b} \\
5 \mathrm{a}, 6,8,19,33\end{array}$ & 3 & 0 & 0 & 0 & 3 & 3 & 57.9 \\
\hline Bg76-1 & $\begin{array}{r}\text { Lankao, } \\
\text { Henan }\end{array}$ & $\begin{array}{l}\text { 5e,6,21,24,46/1a, } 1 \mathrm{c}, 2 \\
3,3 \mathrm{~b}, 3 \mathrm{c}, 3 \mathrm{~g}, 4 \mathrm{a}, 4 \mathrm{~b} \\
5 \mathrm{a}, 7,8,13,19,33\end{array}$ & 3 & 0 & 4 & 0 & 4 & 4 & 71.4 \\
\hline Bg76-3 & $\begin{array}{r}\text { Lankao, } \\
\text { Henan }\end{array}$ & $\begin{array}{l}1 \mathrm{a}, 1 \mathrm{c}, 2,3,3 \mathrm{~b}, 3 \mathrm{~g}, 4 \mathrm{a} \\
4 \mathrm{~b}, 5 \mathrm{e}, 19,21,24,33 \\
46 / 3 \mathrm{c}, 5 \mathrm{a}, 6,8,13\end{array}$ & 0 & 0 & 0 & 0 & 3 & 3 & 26.3 \\
\hline Bg77-1 & $\begin{array}{l}\text { Xihua, } \\
\text { Henan }\end{array}$ & $\begin{array}{l}3 \mathrm{~b}, 3 \mathrm{c}, 5 \mathrm{a}, 5 \mathrm{e}, 13,17 \\
21,24,46 / 1 \mathrm{a}, 1 \mathrm{c}, 2,3,3 \mathrm{~g}, 4 \mathrm{a}, \\
\text { 4b,6,7,8,19,33 }\end{array}$ & 3 & 1 & 3 & 1 & 3 & 4 & 47.6 \\
\hline Bg77-3 & $\begin{array}{l}\text { Xihua, } \\
\text { Henan }\end{array}$ & $\begin{array}{l}\text { 1a, } 1 \mathrm{c}, 2,5 \mathrm{e}, 13,17 \\
21,24 / 3,3 \mathrm{~b}, 3 \mathrm{c}, 3 \mathrm{~g}, 4 \mathrm{a} \\
\text { 4b,5a,6,7,8,19,33 }\end{array}$ & 0 & 0 & 0 & ND & 3 & 3 & 60.0 \\
\hline
\end{tabular}


Table 1. (continued from preceding page)

\begin{tabular}{|c|c|c|c|c|c|c|c|c|c|}
\hline Isolates & Origin & $\begin{array}{l}\text { Avirulent/virulent } \\
\text { on } P m \text { genes }\end{array}$ & $\begin{array}{c}\text { Liangxing } 66 \\
\text { (PmLX66) }\end{array}$ & $\begin{array}{l}\text { Wennong } 14 \\
\quad(P m W 14)\end{array}$ & $\begin{array}{c}\text { Ulka/8*Cc } \\
\quad(P m 2)\end{array}$ & $\begin{array}{c}\text { Tabasco } \\
(P m 48)\end{array}$ & Chancellor & $\begin{array}{c}\text { Zhongzuo } \\
9504\end{array}$ & $\begin{array}{c}\text { Virulence } \\
\text { frequency }(\%)\end{array}$ \\
\hline Bg78-1 & $\begin{array}{l}\text { Xinxiang, } \\
\text { Henan }\end{array}$ & $\begin{array}{l}\text { 1a, } 1 \mathrm{c}, 2,3,3 \mathrm{~b}, 5 \mathrm{e}, 6 \\
21,24,46 / 3 \mathrm{c}, 3 \mathrm{~g}, 4 \mathrm{a}, 4 \mathrm{~b}, 5 \mathrm{a}, \\
7,8,13,17,19,33\end{array}$ & 0 & 0 & 0 & 0 & 4 & 4 & 52.4 \\
\hline Bg78-2 & $\begin{array}{l}\text { Xinxiang, } \\
\text { Henan }\end{array}$ & $\begin{array}{l}1 \mathrm{a}, 1 \mathrm{c}, 2,5 \mathrm{e}, 8,13,21 \\
24 / 3,3 \mathrm{~b}, 3 \mathrm{c}, 3 \mathrm{~g}, 4 \mathrm{a}, 4 \mathrm{~b}, 5 \mathrm{a} \\
6,7,17,19,33\end{array}$ & 0 & 0 & 1 & 0 & 3 & 3 & 61.9 \\
\hline Bg78-3 & $\begin{array}{l}\text { Xinxiang, } \\
\text { Henan }\end{array}$ & $\begin{array}{l}1 \mathrm{a}, 1 \mathrm{c}, 2,3,3 \mathrm{~b}, 3 \mathrm{c}, 3 \mathrm{~g}, 4 \mathrm{a}, 4 \mathrm{~b} \\
\text { 5a,5e,6,13,17,19,21 } \\
24,33,46 / 8\end{array}$ & 0 & 0 & 0 & 0 & 3 & 3 & 9.5 \\
\hline Bg68-1 & Beijing & $\begin{array}{c}1 \mathrm{a}, 1 \mathrm{c}, 2,3 \mathrm{~b}, 3 \mathrm{c}, 4 \mathrm{a}, 4 \mathrm{~b} \\
5 \mathrm{e}, 6,13,17,21,24,33 \\
46 / 3,3 \mathrm{~g}, 5 \mathrm{a}, 7,8,19\end{array}$ & 0 & 0 & 0 & 0 & 3 & 3 & 28.6 \\
\hline Bg68-2 & Beijing & $\begin{array}{l}1 \mathrm{a}, 1 \mathrm{c}, 2,4 \mathrm{a}, 4 \mathrm{~b}, 5 \mathrm{e}, 6,13,21 \\
24,33,46 / 3,3 \mathrm{~b}, 3 \mathrm{c}, 3 \mathrm{~g}, 5 \mathrm{a} \\
7,8,19\end{array}$ & 0 & 0 & 0 & 0 & 3 & 4 & 40.0 \\
\hline Bg68-3 & Beijing & $\begin{array}{l}\text { 1a, } 1 \mathrm{c}, 2,3 \mathrm{~b}, 4 \mathrm{a}, 5 \mathrm{a}, 5 \mathrm{e}, 6 \\
13,21,24,33,46 / 3,3 \mathrm{c}, 3 \mathrm{~g} \\
4 \mathrm{~b}, 7,8,17,19\end{array}$ & 0 & 0 & 0 & 0 & 3 & 3 & 47.6 \\
\hline Bg87 & Beijing & $\begin{array}{l}1 \mathrm{a}, 1 \mathrm{c}, 2,3 \mathrm{~b}, 4 \mathrm{a}, 4 \mathrm{~b}, 5 \mathrm{e}, 13 \\
21,24,33,46 / 3,3 \mathrm{c}, 3 \mathrm{~g}, 5 \mathrm{a} \\
6,7,8,17,19\end{array}$ & 0 & 0 & 0 & 0 & 3 & 4 & 47.6 \\
\hline Bg91-2 & Beijing & $\begin{array}{c}1 \mathrm{a}, 1 \mathrm{c}, 5 \mathrm{e} / 2,3,3 \mathrm{~b}, 3 \mathrm{c}, 3 \mathrm{~g} \\
4 \mathrm{a}, 4 \mathrm{~b}, 5 \mathrm{a}, 6,7,8 \\
17,19,21,24,33\end{array}$ & 0 & 3 & 4 & ND & 4 & 4 & 84.2 \\
\hline Bg86-1 & $\begin{array}{l}\text { Yangzhou, } \\
\text { Jiangsu }\end{array}$ & $\begin{array}{l}1 \mathrm{a}, 1 \mathrm{c}, 2,5 \mathrm{e}, 8,13,21,24 \\
33,46 / 3,3 \mathrm{~b}, 3 \mathrm{c}, 3 \mathrm{~g}, 4 \mathrm{a}, 4 \mathrm{~b}, \\
5 \mathrm{a}, 7,17,19\end{array}$ & 0 & 0 & 0 & 0 & 3 & 4 & 50.0 \\
\hline Bg86-2 & $\begin{array}{c}\text { Yangzhou, } \\
\text { Jiangsu }\end{array}$ & $\begin{array}{l}1 \mathrm{c}, 2,3 \mathrm{~b}, 5 \mathrm{e}, 13,21 \\
24,33,46 / 1 \mathrm{a}, 3,3 \mathrm{c}, 3 \mathrm{~g}, 4 \mathrm{a} \\
\text { 4b,5a,7,8,17,19 }\end{array}$ & 3 & 0 & 0 & 2 & 3 & 4 & 55.0 \\
\hline Bg86-3 & $\begin{array}{l}\text { Yangzhou, } \\
\text { Jiangsu }\end{array}$ & $\begin{array}{l}1 \mathrm{a}, 1 \mathrm{c}, 2,3,4 \mathrm{a}, 4 \mathrm{~b}, 6 \\
17,21,24,33,46 / 3 \mathrm{~b}, 3 \mathrm{c}, 3 \mathrm{~g} \\
5 \mathrm{a}, 5 \mathrm{e}, 7,8,13,19\end{array}$ & 0 & 0 & 0 & 0 & 3 & 4 & 42.9 \\
\hline Bg89-1 & $\begin{array}{l}\text { Wenjiang, } \\
\text { Sichuan }\end{array}$ & $\begin{array}{l}\text { 1a,1c,2,3,4b,5e, } \\
\text { 21/3b,3c,3g,4a,5a,6, } \\
7,8,17,19,24,33\end{array}$ & 2 & 3 & 0 & ND & 3 & 4 & 68.4 \\
\hline Bg90-1 & $\begin{array}{l}\text { Wenjiang, } \\
\text { Sichuan }\end{array}$ & $\begin{array}{l}1 \mathrm{a}, 1 \mathrm{c}, 2,5 \mathrm{e}, 7,17 \\
21 / 3,3 \mathrm{~b}, 3 \mathrm{c}, 3 \mathrm{~g}, 4 \mathrm{a}, 4 \mathrm{~b}, 5 \mathrm{a} \\
6,8,19,24,33\end{array}$ & 0 & 0 & 0 & ND & 3 & 4 & 63.2 \\
\hline
\end{tabular}

the Wennong Seed Co. Ltd., Tai'an, Shandong, has been commercialized in the northern part of the Yellow and Huai River Valleys winter wheat zone (Chen and Sun 2012) and the provincial level in Shandong (Sun et al. 2011). Wheat line Ulka/8*Cc, with Pm2 (Briggle 1969), and the German wheat 'Tabasco', carrying gene Pm48 (which was redesignated from $P m 46$ ) (Gao et al. 2012), were used for comparison purposes.

A set of 21 wheat genotypes that carry single $P m$ genes was used to determine the avirulence or virulence arrays of $B$. graminis $\mathrm{f}$. sp. tritici isolates. Chancellor and 'Zhongzuo 9504' wheat were used as susceptible controls and the latter was also used to maintain and increase the $B$. graminis $\mathrm{f}$. sp. tritic $i$ isolates. To determine allelic relationships, Liangxing 66 , Wennong 14 , and $\mathrm{Ulka} / 8 * \mathrm{Cc}$ were intercrossed to produce segregating $\mathrm{F}_{2}$ populations.

B. graminis f. sp. tritici isolates. Sixty $B$. graminis f. sp. tritici isolates were collected from fields across different parts of northern China and used to test lines with PmLX66, PmW14, Pm2, and Pm48 (Table 1). Single-pustule progeny of each isolate were made three times prior to disease assessments. Isolates were maintained on seedlings of Zhongzuo 9504. In a previous study, the resistance to B. graminis f. sp. tritici isolate E20 (avirulence/virulence formula: Pmlc, Pm2, Pm4a, Pm4b, Pm4c, Pm5e, Pm12, Pm13, Pm16, Pm20, Pm21, Pm24, Pm33, and Pm43/Pmla, Pm3a, Pm3b, Pm3c, Pm3g, Pm5a, Pm6, Pm7, Pm8, Pm17, Pm19, and Pm40) (Zhao et al. 2013) was controlled by single dominant genes in Liangxing 66 and Wennong 14 as demonstrated with the $\mathrm{F}_{2}$ segregating populations derived from the crosses between resistant Liangxing 66 and Wennong 14 and susceptible 'Jingshuang 16' (Song et al. 2012). Thus, isolate E20 was used to inoculate the $\mathrm{F}_{2}$ populations in the allelism tests.

Molecular marker analysis. Total genomic DNA was extracted from young seedling leaves using the cetyletrimethylammonium bromide protocol (Saghai-Maroof et al. 1984). Sequence-characterized amplified region (SCAR) marker SCAR203 (forward: 5' TAAACCCTGAGAATGCAG-3', reverse: 5'-GAGACCATGA GACCCACC-3') and simple-sequence repeat (SSR) marker $X c f d 81$ (forward: 5'-TATCCCCAATCCCCTCTTTC-3', reverse: 5' GTCAATTGTGGCTTGTCCCT-3'), closely linked to PmLX66, PmW14, and Pm2 (Li et al. 2009; Huang et al. 2012; Song et al. 2014), were used to examine Liangxing 66, Wennong 14, and Ulka/ $8 * \mathrm{Cc}$. Each reaction mixture $(10 \mu \mathrm{l})$ consisted of $20 \mathrm{ng}$ of template DNA, $0.1 \mu \mathrm{M}$ each primer, $0.2 \mathrm{mM}$ dNTPs, $1 \times$ polymerase chain reaction (PCR) buffer, $1.5 \mathrm{mM} \mathrm{MgCl}_{2}$, and $1 \mathrm{U}$ of $T a q$ DNA polymerase. Amplification of DNA was carried out in a Biometra T3000 Thermocycler (ABL, New York) with the following conditions: $94^{\circ} \mathrm{C}$ for $4 \mathrm{~min}$, followed by 38 cycles of $94^{\circ} \mathrm{C}$ for $45 \mathrm{~s}, 55^{\circ} \mathrm{C}$ for $45 \mathrm{~s}$, and $72^{\circ} \mathrm{C}$ for $1 \mathrm{~min}$. The reaction was terminated after a final extension at $72^{\circ} \mathrm{C}$ for $10 \mathrm{~min}$ and then maintained at $4^{\circ} \mathrm{C}$. The PCR products were separated on $8 \%$ nondenatured polyacrylamide gels with a 29:1 ratio of acrylamide and bisacrylamide, and visualized by silver staining.

Allelism tests. Seedlings of the $\mathrm{F}_{2}$ populations were inoculated with the $B$. graminis f. sp. tritici isolate E20 when the first leaves were fully unfolded by brushing the conidiospores from susceptible seedlings of Zhongzuo 9504. Approximately 15 day after inoculation, infection types (IT) on the primary leaf of each plant was scored on a scale of 0 to 4 . 


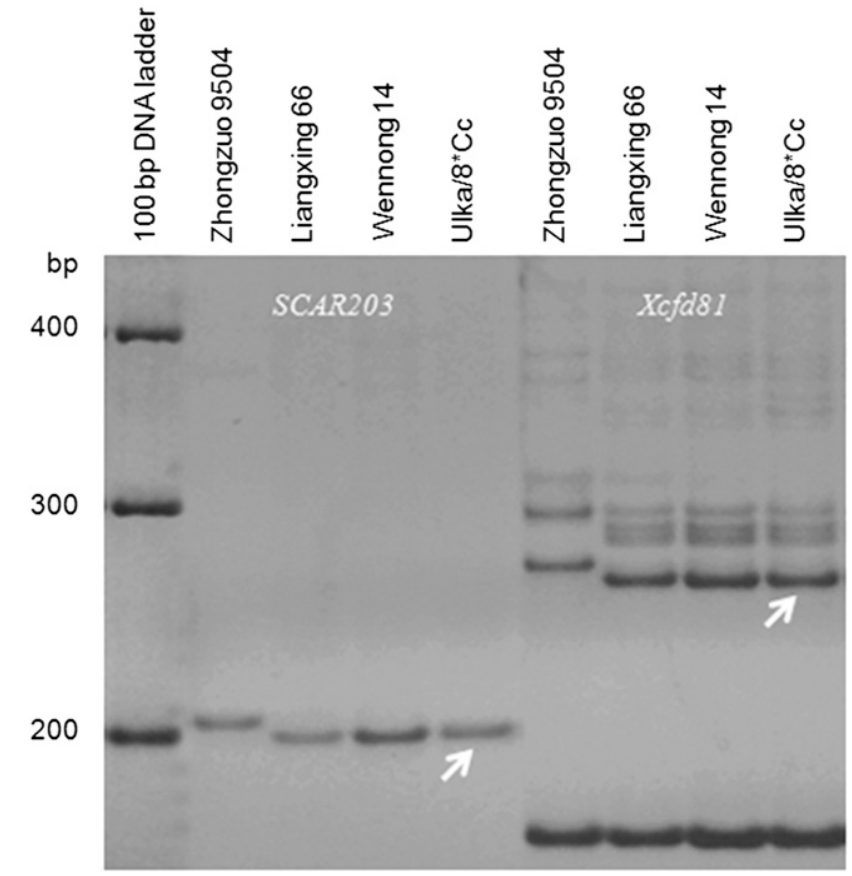

Fig. 1. Amplification profile by markers SCAR203 and Xcfd81 from Liangxing 66, Wennong 14, Ulka/8* $\mathrm{Cc}$, and Zhongzuo 9504. Arrow indicates the target bands specific for $P m 2$.

Table 2. Analysis of allelism relationships between powdery mildew resistance genes PmLX66, PmW14, and Pm2 when tested against Blumeria graminis f. sp. tritici isolate $\mathrm{E} 20$ in the $\mathrm{F}_{2}$ populations derived from Liangxing 66 (PmLX66), Wennong 14 (PmW14), and Ulka/8*Cc (Pm2)

\begin{tabular}{lcrrc}
\hline & & \multicolumn{3}{c}{ Infection type } \\
\cline { 3 - 5 } Cultivar or cross & $\boldsymbol{N}^{\mathbf{z}}$ & $\mathbf{0}$ & $\mathbf{1}$ & $\mathbf{3}$ or 4 \\
\hline Liangxing 66 $($ PmLX66) & 20 & 20 & 0 & $\ldots$ \\
Wennong 14 (PmW14) & 20 & 20 & 0 & $\ldots$ \\
Ulka/8*Cc $(P m 2)$ & 20 & 0 & 20 & $\ldots$ \\
Zhongzuo 9504 (control) & 20 & 0 & 0 & 20 \\
Liangxing 66 $\times$ Ulka/8*Cc & 1,344 & 1,124 & 220 & 0 \\
& 2,275 & 1,955 & 320 & 0 \\
Wennong14 $\times$ Ulka/8*Cc & 2,072 & 1,968 & 104 & 0 \\
& 4,113 & 3,128 & 985 & 0 \\
Liangxing 66 $\times$ Wennong 14 & 1,571 & 1,507 & 64 & 0 \\
& 4,282 & 3,770 & 512 & 0 \\
\hline
\end{tabular}

$\mathrm{z}$ Total number of plants.
Reaction to $B$. graminis f. sp. tritici isolates. A detached-leaf method was used to compare reactions to different $B$. graminis $\mathrm{f}$. sp. tritici isolates in Liangxing 66 (PmLX66), Wennong 14 (PmW14), Ulka/8*Cc (Pm2), and Tabasco (Pm48) (Limpert et al. 1988). Three leaf segments from different seedlings of each genotype were embedded in media on plates containing agar at $6 \mathrm{~g} \mathrm{liter}^{-1}$ supplemented with benzimidazole at $50 \mathrm{mg} \operatorname{liter}^{-1}$ and inoculated separately with $B$. graminis f. sp. tritici isolates by dusting conidiospores on susceptible seedlings of Zhongzuo 9504 prior to incubation at 18 to $21^{\circ} \mathrm{C}$ in a growth cabinet.

IT on each leaf segment was scored on a 0 -to- 4 scale, where $0=$ no visible symptom, $0 ;=$ necrotic flecks, $1=$ necrosis with low sporulation, $2=$ necrosis with moderate sporulation, $3=$ no necrosis with moderate to high sporulation, and $4=$ no necrosis with full sporulation, and where 0 is immune and 4 represents heavy sporulation (Xiao et al. 2013). The phenotype of each wheat line to each isolate was categorized as either resistant (IT 0 to 2 ) or susceptible (IT 3 to 4 ) and the experiment was conducted twice.

\section{Results}

Molecular marker analysis of the segregating populations. SCAR marker SCAR203 and SSR marker $X c f d 81$, which were tightly linked to PmLX66, PmW14, and Pm2, amplified the same band from Liangxing 66, Wennong 14 , and Ulka/ $8 * \mathrm{Cc}$ (Fig. 1). These bands were slightly smaller than that from susceptible Zhongzuo 9504. $S C A R 203$ was used to confirm the genotypes of selected $\mathrm{F}_{2}$ segregating populations used in allelism tests. The amplified fragment specific to $P m 2$ was present in 64, 70, and 82 random $F_{2}$ plants derived from the crosses Liangxing $66 \times$ Ulka/ $/ * \mathrm{Cc}$, Wennong $14 \times \mathrm{Ulka} / 8 * \mathrm{Cc}$, and Liangxing $66 \times$ Wennong 14 , respectively (data not shown).

Allelism test of PmLX66, PmW14, and Pm2. When inoculated with $B$. graminis f. sp. tritici isolate E20, Liangxing 66 and Wennong 14 showed a resistant reaction with IT 0 , whereas Ulka/ $8 * \mathrm{Cc}$ produced IT 1. To determine the allelic relationship between PmLX66, $P m W 14$, and $P m 2$, phenotypes of the $\mathrm{F}_{2}$ plants derived from the crosses between these wheat genotypes were determined in two separate tests using $B$. graminis f. sp. tritici isolate E20 (Table 2). In the first test, $1,344 \mathrm{~F}_{2}$ plants from the cross Liangxing $66 \times \mathrm{Ulka} / 8 * \mathrm{Cc}$ produced 1,124 plants with IT 0 and 220 plants with IT 1 . In the second test, all of the $2,275 \mathrm{~F}_{2}$ plants from this cross had a resistant phenotype (IT 0 or 1 ) and none was susceptible. Among the 2,072 plants from the cross Wennong $14 \times \mathrm{Ulka} / 8^{*} \mathrm{Cc}$ in the first test, 1,968 plants produced IT 0 and 104 plants had IT 1 . None of them was susceptible. In the second test, all of the $4,113 \mathrm{~F}_{2}$ plants from this cross had IT 0 or 1 . In total, $5,853 \mathrm{~F}_{2}$ plants originating from the cross Liangxing $66 \times$ Wennong 14 were tested against $B$. graminis $\mathrm{f}$. sp. tritici isolate E20 in two separate tests. Most plants produced IT 0 and a small proportion of plants had IT 1. No plants were found to have a susceptible

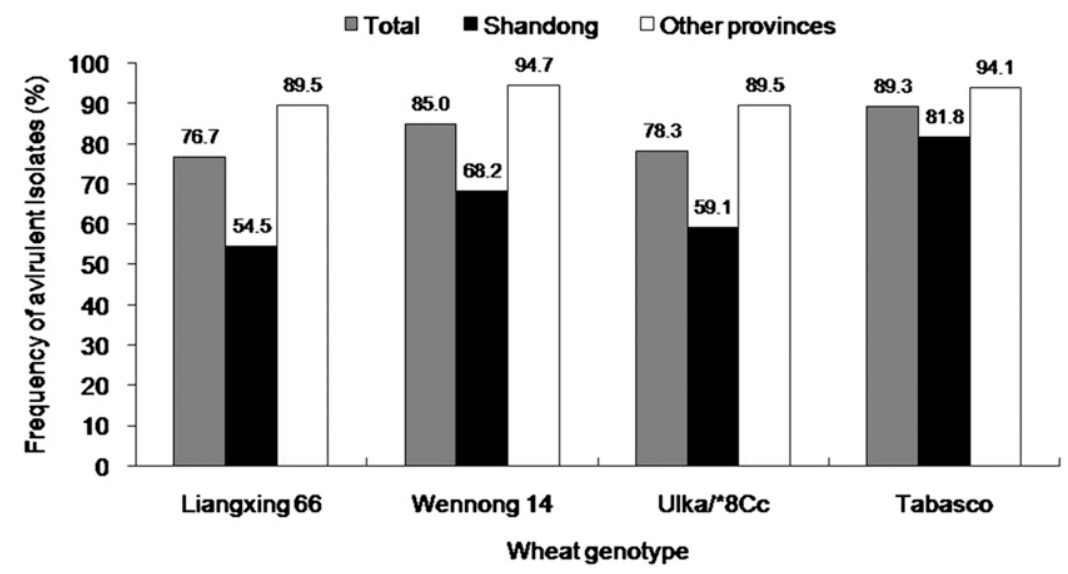

Fig. 2. Frequency of isolates from Shandong and other provinces in China that were avirulent on Liangxing $66(P m L X 66)$, Wennong $14(P m W 14)$, Ulka/8*Cc $(P m 2)$, and Tabasco (Pm48). 
phenotype. In total, none of the $15,657 \mathrm{~F}_{2}$ plants from the three crosses examined was susceptible to isolate E20. These results indicate that genes PmLX66 and PmW14 in Liangxing 66 and Wennong 14, respectively, were allelic to $P m 2$ in Ulka/8*Cc, and $P L X 66$ and $P m W 14$ are also allelic with each other.

Reactions of Liangxing 66, Wennong 14 , Ulka/8*Ce, and Tabasco to B. graminis f. sp. tritici. Sixty isolates were inoculated separately to wheat lines carrying 21 different $P m$ genes and the susceptible controls Chancellor and Zhongzuo 9504 (Table 1). A small percentage of isolates (approximately 1.7 to 5.0\%) were virulent on cultivars possessing Pmlc, Pm21, and Pm24. The percentage of isolates that were virulent on cultivars carrying Pm5e and $P m 13$ were 13.3 and $16.1 \%$, respectively. Over $80 \%$ of isolates were virulent on cultivars carrying $P m 3 c, P m 3 g, P m 5, P m 7, P m 8$, and $P m 19$. A range of 46.7 to $76.7 \%$ of isolates were virulent on cultivars with Pm3, Pm3b, Pm4a, Pm4b, Pm6, Pm17, and Pm33. Isolates Bg91-2 (Beijing) was virulent on all genes except $P m 1 c$ and $P m 5 e$, isolate Bg78-3 (Xinxiang, Henan province) was avirulent on all genes except $P m 7$ and $P m 8$, and isolate Bg82-3 (Shoushan, Shandong province) was avirulent on all the genes except Pml, Pm8, and Pm19 (Table 1). All the $B$. graminis f. sp. tritici isolates were virulent on the susceptible controls Chancellor and Zhongzuo 9504, with IT 3 or 4.

The avirulence frequencies of isolates on Liangxing 66 (PmLX66), Wennong 14 (PmW14), Ulka/8*Cc (Pm2), and Tabasco (Pm48) were $76.7 \%$ (46/60 isolates), $85.0 \%$ (51/60 isolates), $78.3 \%$ (47/60 isolates), and 89.3\% (50/56 isolates), respectively (Fig. 2). Both Liangxing 66 and Wennong 14 were developed in Shandong province; therefore, $22 B$. graminis f. sp. tritici isolates collected from that province were tested. Among them, the frequencies of $B$. graminis f. sp. tritici isolates from Shandong province that were avirulent on Liangxing 66, Wennong 14 , Ulka/8*Cc, and Tabasco were 54.5, $68.2,59.1$, and $81.8 \%$, respectively. These wheat genotypes were even more effective against the $B$. graminis $\mathrm{f}$. sp. tritici isolates from other provinces than those from Shandong province.

Among the 60 B. graminis f. sp. tritici isolates examined, Liangxing 66 produced the same infection types as Ulka/ $8 * \mathrm{Cc}$ in reaction to 51 isolates but differed from $\mathrm{Ulka} / 8 * \mathrm{Cc}$ in reaction to 9 isolates (Table 1). Four isolates were avirulent on Liangxing 66 (IT 0 or 1) but virulent on Ulka/ $8 * \mathrm{Cc}$ (IT 3 or 4 ), and five isolates were virulent on Liangxing 66 (IT 3 or 4 ) but avirulent on Ulka/8*Cc (IT 0 ). Liangxing 66 displayed the same phenotypes as Tabasco in reaction to 44 B. graminis $\mathrm{f}$. sp. tritici isolates out of 56 isolates tested. These two cultivars exhibited different IT in response to 12 isolates, and Tabasco was resistant to all except 2 isolates.

Wennong 14 differed from Ulka/ $8 * \mathrm{Cc}$ in reaction to $12 \mathrm{~B}$. graminis $\mathrm{f}$. sp. tritici isolates. Seven isolates were avirulent on Wennong 14 but virulent on $\mathrm{Ulka} / 8^{*} \mathrm{Cc}$. Isolate $\mathrm{Bg} 77-1$ resulted in IT 1 on Wennong 14 but IT 3 on Ulka/ $8 * \mathrm{Cc}$. In contrast, Wennong 14 and Ulka/ $8 * \mathrm{Cc}$ had IT 3 and 0 , respectively, in their reactions to four isolates. Among the eight $B$. graminis $\mathrm{f}$. sp. tritici isolates, which had different infection types between Wennong 14 and Tabasco, three isolates exhibited IT 0 and 3, while five isolates produced IT 3 and 0 on Wennong 14 and Tabasco, respectively.

Liangxing 66 and Wennong 14 differed in their reactions to $11 \mathrm{~B}$. graminis f. sp. tritici isolates. Wennong 14 was resistant to 8 of 11 isolates (IT 0 or 1) but susceptible to 3 isolates (IT 3 or 4). However, Liangxing 66 was susceptible to all of these $B$. graminis f. sp. tritici isolates except for isolates $\mathrm{Bg} 84-1, \mathrm{Bg} 89-1$, and $\mathrm{Bg} 91-2$. Of 56 isolates examined, Liangxing 66 and Wennong 14 were different from Tabasco in the reactions to 13 and 8 isolates, respectively. In addition, isolate $\mathrm{Bg} 44-5$ originating from Zhanhua, Shandong province, was virulent, whereas 37 isolates were avirulent on the four genotypes.

\section{Discussion}

Previous studies have demonstrated that the dominant genes PmLX66 and PmW14 were present in Liangxing 66 and Wennong 14 and molecular marker analyses indicated that PmLX66 and PmW14 shared the same locus as Pm2 on chromosome 5DS on the basis of linked markers Xcfd81 and SCAR203. Allelism tests carried out in the present work indicated that the three genes were allelic.
Given the different responses to various B. graminis f. sp. tritici isolates found in the present work as well as in earlier studies (Huang et al. 2012; Song et al. 2014), PmLX66 and PmW14 must be new alleles of Pm2 locus.

In addition to Liangxing 66 and Wennong 14, Pm genes on chromosome 5DS have been identified in several Chinese wheat lines; for example, 'D57' (Ma et al. 2011), 'Nongda 399' (Li et al. 2013), and 'X3986-2' (Ma et al. 2014), as well British 'Brock' wheat (Li et al. 2009; Lillemo et al. 2010). The close linkages between these powdery mildew resistance genes and Pm2 was established based on common linked molecular markers such as $X c f d 81$. Study of a cross between Tabasco and Ulka/ $8 * \mathrm{Cc}$ showed that the gene $P m 48$ in Tabasco was different from $P m 2$ despite their common molecular marker Xcfd81 (Gao et al. 2012).

$P m 2$ has been widely utilized in breeding programs in China and Europe (Bennett 1984; Liu et al. 2000). High virulence frequencies for Pm2 in B. graminis f. sp. tritici isolates were reported in some areas of the United States (Niewoehner and Leath 1998; Parks et al. 2008; Persaud and Lipps 1995) and China (Shi et al. 2009). In a recent study, the virulence frequency in $B$. graminis f. sp. tritici isolates from different regions of China on Ulka/ $8 * \mathrm{Cc}$ varied from $31.8 \%$ (north China) to $58.3 \%$ (Xinjiang) (Zeng et al. 2014). In previous studies, Liangxing 66 and Wennong 14 were resistant to approximately $60 \%$ (25 of 42) and $82.7 \%$ (43 of 52) of isolates, respectively, from northern China (Huang et al. 2012; Song et al. 2014). In this study, Liangxing 66 and Wennong 14 conferred comparable or slightly higher frequencies and different arrays of resistance compared with Ulka/ $8 * \mathrm{Cc}$ to a set of $60 \mathrm{~B}$. graminis $\mathrm{f}$. $\mathrm{sp}$. tritici isolates from different parts of China. Additionally, Liangxing 66 and Wennong 14 are commercial cultivars and more adaptive to the local environments than $\mathrm{Ulka} / 8 * \mathrm{Cc}$.

In summary, genes PmLX66 and PmW14 in Liangxing 66 and Wennong 14 winter wheat, previously mapped on chromosome 5DS, were effective against over $70 \%$ of the $B$. graminis f. sp. tritici isolates originating from wheat-producing regions in China where they are commercialized. These genes confer different reaction patterns in response to $B$. graminis f. $\mathrm{sp}$. tritici isolates compared with the $P m 2$ gene derived from Ulka, with which they are allelic. Because Liangxing 66 and Wennong 14 are widely grown commercial winter wheat cultivars and free of linkage drag, the identification of genes for resistance to powdery mildew will facilitate their application in developing disease-resistant cultivars or in combination with other powdery mildew resistance genes for effectively controlling the disease in wheat production.

\section{Acknowledgments}

We thank T. D. Murray, Department of Plant Pathology, Washington State University, for his critical review of the manuscript; S. B. Cai, Jiangsu Academy of Agricultural Science, for providing seed of Tabasco; and the National Natural Science Foundation (31471491), Ministry of Science and Technology (2014ZX0800906B-003), Ministry of Agriculture (CARS-3-1-2), and a CAAS Innovation Team Project for their financial support

\section{Literature Cited}

Bennett, F. G. A. 1984. Resistance to powdery mildew in wheat: A review of its use in agriculture and breeding programmes. Plant Pathol. 33:279-300.

Briggle, L. W. 1969. Near-isogenic lines of wheat with genes for resistance to Erysiphe graminis f. sp. tritici. Crop Sci. 9:70-72.

Cao, X. R., Zhou, Y. L., Duan, X. Y., Song, Y. L., He, W. L., Ding, K. J., Wang, B. T., and Xia, X. C. 2010. Postulation of wheat powdery mildew resistance genes in 101 wheat cultivars (lines) from major wheat regions in China. J. Triticeae Crops 30:948-953.

Chen, Y. L., and Sun, K. 2012. New wheat cultivar Wennong 14. China Seed Ind. $3: 61$.

Cowger, C., Miranda, L., Griffey, C., Hall, M., Murphy, J. P., and Maxwell, J. 2012. Wheat powdery mildew. Pages 84-119 in: Disease Resistance in Wheat. I. Sharma, ed. CABI, Oxfordshire.

Gao, H. D., Zhu, F. F., Jiang, Y. J., Wu, J. Z., Yan, W., Zhang, Q. F., Jacobi, A., and Cai, S. B. 2012. Genetic analysis and molecular mapping of a new powdery mildew resistance gene Pm46 in common wheat. Theor. Appl. Genet. 125: 967-973.

Hsam, S. L. K., and Zeller, F. J. 1997. Evidence of allelism between genes Pm8 and Pm17 and chromosomal location of powdery mildew and leaf rust resistance genes in the common wheat cultivar Amigo. Plant Breed. 116:119-122. 
Huang, J., Zhao, Z. H., Song, F. J., Wang, X. M., Xu, H. X., Huang, Y., An, D. G., and $\mathrm{Li}, \mathrm{H}$. J. 2012. Molecular detection of a gene effective against powdery mildew in the wheat cultivar Liangxing 66. Mol. Breed. 30:1737-1745.

Huang, X. Q., Wang, L. X., Xu, M. X., and Röder, M. S. 2003. Microsatellite mapping of the powdery mildew resistance gene Pm5e in common wheat (Triticum aestivum L.). Theor. Appl. Genet. 106:858-865.

Hulbert, S. H., Webb, C. A., Smith, S. M., and Sun, Q. 2001. Resistance gene complexes: Evolution and utilization. Annu. Rev. Phytopathol. 39:285-312.

Li, D., Yuan, C. G., Wu, H. B., Zhang, D., Liang, Y., Wang, Z. Z., Wu, Q. H., Chen, Y. X., Yang, Z. M., Sun, Q. X., and Liu, Z. Y. 2013. SSR and AFLPderived SCAR markers associated with the powdery mildew resistance gene in common wheat cultivar ND399. J. Plant Genet. Resour. 14:104-108.

Li, G. Q., Fang, T. L., Zhu, J., Gao, L. L., Li, S., Xie, C. J., Yang, Z. M., Sun, Q. X., and Liu, Z. Y. 2009. Molecular identification of a powdery mildew resistance gene from common wheat cultivar Brock. Acta Agron. Sin. 35:1613-1619.

Li, H. J., Wang, X. M., Song, F. J., Wu, C. P., Wu, X. F., Zhang, N., Zhou, Y., and Zhang, X. Y. 2011. Response to powdery mildew and detection of resistance genes in wheat cultivars from China. Acta Agron. Sin. 37:943-954.

Lillemo, M., Skinnes, H., and Brown, J. K. M. 2010. Race specific resistance to powdery mildew in Scandinavian wheat cultivars, breeding lines and introduced genotypes with partial resistance. Plant Breed. 129:297-303.

Limpert, E., Andrivon, D., and Felsenstein, F. G. 1988. Influence of different benzimidazole concentrations in agar medium on senescence of wheat leaf segments and on growth and sporulation of the wheat powdery mildew pathogen. J. Plant Dis. Prot. 95:301-306.

Liu, J., Liu, D., Tao, W., Li, W., Wang, S., Chen, P., Cheng, S., and Gao, D. 2000. Molecular marker-facilitated pyramiding of different genes for powdery mildew resistance in wheat. Plant Breed. 119:21-24.

Lutz, J., Katzhammer, M., Stephan, U., Felsenstein, F., Oppitz, K., and Zeller, F. 1995. Identification of powdery mildew resistance genes in common wheat (Triticum aestivum L.). V. Old German cultivars and cultivars released in the former GDR. Plant Breed. 114:29-33.

Lutz, J., Limpert, E., Bartos, P., and Zeller, F. 1992. Identification of powdery mildew resistance genes in common wheat (Triticum aestivum L.). I. Czechoslovakian cultivars. Plant Breed. 108:33-39.

Ma, H. Q., Kong, Z. X., Fu, B. S., Li, N., Zhang, L. X., Jia, H. Y., and Ma, Z. Q. 2011. Identification and mapping of a new powdery mildew resistance gene on chromosome 6D of common wheat. Theor. Appl. Genet. 123:1099-1106.

Ma, P. T., Xu, H. X., Luo, Q. L., Qie, Y. M., Zhou, Y. L., Xu, Y. F., Han, H. M., Li, L. H., and An, D. G. 2014. Inheritance and genetic mapping of a gene for seedling resistance to powdery mildew in wheat line X3986-2. Euphytica 200:149-157.

Niewoehner, A. S., and Leath, S. 1998. Virulence of Blumeria graminis f. sp. tritici on winter wheat in the Eastern United States. Plant Dis. 82:64-68.

Parks, R., Carbone, I., Murphy, J., Marshall, D., and Cowger, C. 2008. Virulence structure of the eastern U.S. wheat powdery mildew population. Plant Dis. 92:1074-1082.

Persaud, R., and Lipps, P. 1995. Virulence genes and virulence gene frequencies of Blumeria graminis f. sp. tritici in Ohio. Plant Dis. 79:494-499.

Pugsley, A. T., and Carter, M. V. 1953. The resistance of twelve varieties of Triticum vulgare to Erysiphe graminis tritici. Aust. J. Biol. Sci. 6:335-346.

Purnhauser, L., Bóna, L., and Láng, L. 2011. Occurrence of 1BL.1RS wheat-rye chromosome translocation and of $\mathrm{Sr} 36 / \mathrm{Pm} 6$ resistance gene cluster in wheat cultivars registered in Hungary. Euphytica 179:287-295.

Rabinovich, S. V. 1998. Importance of wheat-rye translocations for breeding modern cultivars of Triticum aestivum L. Euphytica 100:323-340.
Saghai-Maroof, M. A., Soliman, K. M., Jorgensen, R. A., and Allard, R. W. 1984 Ribosomal DNA spacer-length polymorphisms in barley: Mendelian inheritance, chromosomal location, and population dynamics. Proc. Natl. Acad. Sci. USA 81:8014-8018.

Sang, D. J., Xu, W. G., Hu, L., Dong, H. B., and Wang, Y. S. 2006. The molecular identification of powdery mildew resistance genes in the cultivars in Henan province and application of molecular marker-assisted breeding. Acta Agric. Boreali-Occident Sin. 21:86-91.

Schmolke, M., Mohler, V., Hartl, L., Zeller, F. J., and Hsam, S. L. K. 2012. A new powdery mildew resistance allele at the $\mathrm{Pm} 4$ wheat locus transferred from einkorn (Triticum monococcum). Mol. Breed. 29:449-456.

Shi, Y. Q., Wang, B. T., Li, Q., Wu, X. Y., Wang, F., Liu, H., Tian, Y. E., and Liu, Q. R. 2009. Analysis on the virulent genes of Erysiphe graminis f. sp. tritici and the resistance genes of wheat commercial cultivars in Shaanxi province. J. Triticeae Crops 29:706-711.

Singrün, C. H., Hsam, S. L. K., Hartl, L., Zeller, F. J., and Mohler, V. 2003 Powdery mildew resistance gene Pm22 in cultivar Virest is a member of the complex Pm1 locus in common wheat (Triticum aestivum L. em Thell.) Theor. Appl. Genet. 106:1420-1424.

Song, W., Sun, H. G., Sun, Y. L., Zhao, Z. H., Wang, X. M., Wu, X. F., and Li, H. J. 2014. Chromosomal localization of the gene for resistance to powdery mildew in the wheat cultivar Wennong 14. Acta Agron. Sin. 40:798-804.

Song, F. J., Xiao, M. G., Huang, J., Wang, X. M., Zhu, Z. D., Wu, X. F., and Li, H. J. 2012. Inheritance of resistance to powdery mildew in 12 wheat varieties (lines). Acta Agron. Sin. 38:1339-1345.

Summers, R. W., and Brown, J. K. M. 2013. Constraints on breeding for disease resistance in commercially competitive wheat cultivars. Plant Pathol. 62 (Suppl. 1):115-121

Sun, K., Liu, N., and Zhang, X. S. 2011. Wennong 14: New wheat cultivar with high yield, good quality, and multiple resistances. China Seed Ind. 10:47.

Xiao, M. G., Song, F. J., Jiao, J. F., Wang, X. M., Xu, H. X., and Li, H. J. 2013 Identification of the gene Pm47 on chromosome 7BS conferring resistance to powdery mildew in the Chinese wheat landrace Hongyanglazi. Theor. Appl. Genet. 126:1397-1403.

Xue, F., Wang, C. Y., Li, C., Duan, X. Y., Zhou, Y. L., and Zhao, N. J. 2012 Molecular mapping of a powdery mildew resistance gene in common wheat landrace Baihulu and its allelism with Pm24. Theor. Appl. Genet. 125: $1425-1432$.

Yahiaoui, N., Kaur, N., and Keller, B. 2009. Independent evolution of functional $P m 3$ resistance genes in wild tetraploid wheat and domesticated bread wheat. Plant J. 57:846-856.

Zeng, F. S., Yang, L. J., Gong, S. J., Shi, W. Q., Zhang, X. J., Wang, H., Xiang, L. B., Xue, M. F., and Yu, D. Z. 2014. Virulence and diversity of Blumeria graminis f. sp. tritici population in China. J. Integr. Agric. 13:2424-2437.

Zhan, H. X., Chang, Z. J., Yang, Z. J., Zhang, H. J., and Li, X. 2010. Sources and evaluation of powdery mildew resistance genes in wheat. Chin. Agric. Sci. Bull. 26:42-46.

Zhao, Z. H., Sun, H. G., Song, W., Lu, M., Huang, J., Wu, L. F., Wang, X. M., and Li, H. J. 2013. Genetic analysis and detection of the gene MlLX99 on chromosome $2 \mathrm{BL}$ conferring resistance to powdery mildew in the wheat cultivar Liangxing 99. Theor. Appl. Genet. 126:3081-3089.

Zhou, Y. L., Duan, X. Y., Chen, G., Sheng, B. Q., and Zhang, Y. 2002. Analyses of resistance genes of 40 wheat cultivars or lines to wheat powdery mildew. Acta Phytopathol. Sin. 32:301-305. 